\title{
Optimization of Focused Ion Beam Patterning Parameters for Direct Integration of Plasmonic Nanostructures on Silicon Photodiodes ${ }^{+}$
}

\author{
Elia Scattolo ${ }^{1,2, *}$, Alessandro Cian ${ }^{1}\left(\mathbb{C}\right.$, Damiano Giubertoni ${ }^{1}$, Giovanni Paternoster ${ }^{1}$, Luisa Petti ${ }^{2} \mathbb{C}$ \\ and Paolo Lugli ${ }^{2}$ \\ 1 Sensors and Devices Center, Bruno Kessler Foundation, 38123 Trento, Italy; acian@fbk.eu (A.C.); \\ giuberto@fbk.eu (D.G.); paternoster@fbk.eu (G.P.) \\ 2 Sensing Technology Lab, Faculty of Science and Technology, Free University of Bozen, 39100 Bozen, Italy; \\ luisa.petti@unibz.it (L.P.); paolo.lugli@unibz.it (P.L.) \\ * Correspondence: escattolo@fbk.eu \\ + Presented at the 8th International Electronic Conference on Sensors and Applications, 1-15 November 2021; \\ Available online: https: / / ecsa-8.sciforum.net.
}

check for updates

Citation: Scattolo, E.; Cian, A.;

Giubertoni, D.; Paternoster, G.; Petti, L.; Lugli, P. Optimization of Focused Ion Beam Patterning Parameters for Direct Integration of Plasmonic Nanostructures on Silicon Photodiodes. Eng. Proc. 2021, 10, 2. https://doi.org/10.3390/ecsa-811259

Academic Editor: Stefano Mariani

Published: 1 November 2021

Publisher's Note: MDPI stays neutral with regard to jurisdictional claims in published maps and institutional affiliations.

Copyright: (c) 2021 by the authors. Licensee MDPI, Basel, Switzerland. This article is an open access article distributed under the terms and conditions of the Creative Commons Attribution (CC BY) license (https:// creativecommons.org/licenses/by/ $4.0 /)$.

\begin{abstract}
The possibility of integrating plasmonic nanostructures directly on an active device, such as a silicon photodetector, is a challenging task of interest in many applications. Among the available nanofabrication techniques to realize plasmonic nanostructures, Focused Ion Beam (FIB) is surely the most promising, even if it is characterized by certain limitations, such as ion implantation in the substrate. In this work, we demonstrate the direct integration of plasmonic nanostructures directly on an active Si-photodetector by patterning a silver film with FIB. To avoid ion implantation and to therefore guarantee unaltered device behavior, both the patterning parameters and the geometry of the nanostructures were implemented by Montecarlo and Finite-Difference Time-Domain simulations.
\end{abstract}

Keywords: silicon photodiode; plasmonic nanostructures; focused ion beam

\section{Introduction}

In the last twenty years, many theoretical studies have shown that plasmonic structures have astonishing, unique, and interesting optical characteristics [1-4]. As a direct consequence, many scientific and industrial applications have tried to take advantages of plasmonics, resulting in substantial innovation in nanofabrication methods. As an example, the combination of plasmonic nanostructures with complementary metal-oxidesemiconductor (CMOS) optical sensors that are capable of converting photons into electrical signals have generated great interest due to their potentiality of extending the spectral responsivity of silicon in a wider spectral region from the ultra-violet (UV) region up to the near infra-red (NIR) region [5]. Unlike the fabrication of plasmonic nanostructures on an inert substrate, the production of plasmonic nanostructures on active sensors requires the consideration of not only the surface topography but also the active behaviors of the sensors. Therefore, the choice of the production technique requires special considerations. Focused Ion Beam (FIB), which does not require a mask or a photoresist (as the ion beam is focused directly on the material of interest), is one of the methods that can be used for the fabrication of CMOS technology-compatible nanostructures [6-9]. The main advantage of direct nano-patterning by FIB is the total flexibility that it provides in terms of the shape and aspect ratio. Nevertheless, direct patterning can cause ion implantation in the substrate, causing flaws and modifying the electrical behavior of the sensors [10]. Thus, to fabricate plasmonic nanostructures that are directly on the top of a CMOS sensors is pivotal to tune the FIB process parameters, such as beam energy and current and dose and ions species. In this contribution, we demonstrate the integration of metallic plasmonic nanoarrays that have been developed directly on top of an active silicon photodetector by means of FIB 
patterning, showing no damage to the active behavior of the device. Both the structures and the patterning parameters were optimized by Finite-Difference Time-Domain (FTDT) and Montecarlo simulations, while the unaltered Si-detector behavior was evaluated by current-voltage (I-V) measurements.

\section{Plasmonic Enhancement Optical Sensors}

Among photon detecting systems, single photon avalanche diodes (SPADs) have been studied consistently in recent years, with the purpose of achieving higher detection efficiency and a high timing resolution. Specifically, many applications are interested in having a high time resolution device, such as LiDAR (at 850-950 nm wavelengths), NIR spectroscopy, quantum computation, to exploit near infrared (NIR) and to detect light from NIR-emitting scintillators [11-14]. Differently from silicon photodiodes (PDs), which have a thicker active region that can be employed to achieve higher efficiency in the NIR region, SPADs need thinner active regions for the more technological aspects as well as to those aspects that are related to the time resolution that is required [15]. Hence, the integration of plasmonic metallic nanostructures supporting highly superficial confined light in a thin SPAD is a breakthrough solution to overcome this limitation. Recently, some interesting nanostructures have been investigating (i) 1- and 2-dimensional gratings [16]; (ii) bullseye structures [17]; and (iii) nano-pillars and nano-holes arrays [18]. Among those, in this work, 1-D and 2-D metallic nanogratings are investigated by considering their feasibility and possible integration with a Si-based photodetector and CMOS technologies.

\section{Methods}

The plasmonic nanoarrays were directly integrated on a silicon-based photodiode, i.e., a device that is able to transduce incident light (photons) in measurable currents (electrons). The proposed structure is composed of three parts (excluding the photodetector [5]): dielectric material, metallic grating, and a passivation layer on top, Figure $1 \mathrm{~b}$. The dielectric material has two fundamental aims: (i) the passivation of the surface of the detector and (ii) the guarantee of the excitation of the surface plasmon polaritons (SPPs). Silicon nitride $\left(\mathrm{Si}_{3} \mathrm{~N}_{4}\right)$ and silicon oxide $\left(\mathrm{SiO}_{2}\right)$ are perfect candidates for use as dielectric materials because of their process compatibility with CMOS technology and because of their chemical compatibility with Si-based devices. The optimal thickness for the dielectric layer (which maximizes the enhancement) depends on the material selected, and from the theoretical simulations, for both materials, this was determined to be in the range of 5-20 nm. To fabricate the metal grating, a direct pattern can be employed by FIB after the evaporation of silver by physical vapor deposition (PVD), [19]. After the patterning, a passivation layer (usually PMMA) is spin coated on top of the grating to guarantee performance stability and to avoid metal oxide formation.

The enhancement of the Si-photodetector efficiency coupled with metallic grating is achievable due to the confinement of photons (by the nanostructure) at the interface by the excitation of the SPPs, [20-22]. The wavelength at which the SPPs is excited is a function of grating geometry, dielectric thickness, and passivation thickness, and, therefore, the confinement of the photons is achieved. Moreover, the direct integration of the different metallic plasmonic structures on top of an active device without damaging its behavior is a challenging task. Therefore, before starting the fabrication, both the Montecarlo simulations [23,24] and FDTD [25] simulations were run to tune the ion beam parameters and to optimize the grating geometry, respectively. 

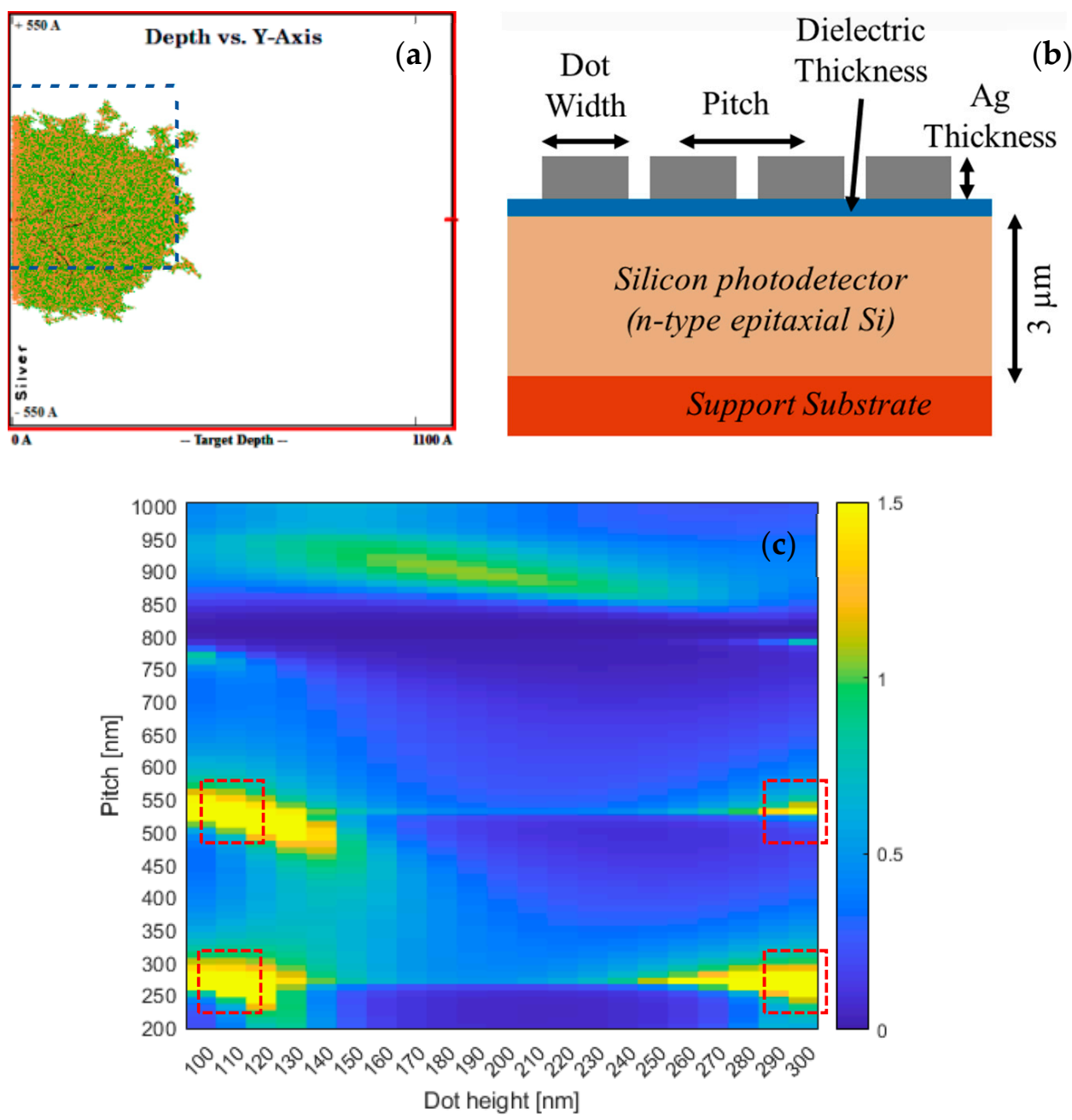

Figure 1. (a) Collision cascade from Montecarlo simulations inside silver layer; (b) schematic cross section of the proposed device, from top: silver nanograting, silicon nitride dielectric and silicon photodetector; (c) finite-difference time-domain [25] of simulated absorption at a wavelength of $950 \mathrm{~nm}$ normalized to the absorption of silicon covered with perfect anti-reflective coating (PARC).

\subsection{Montecarlo Simulations}

As discussed before, Montecarlo simulations are fundamental to tune the ion beam parameters. The aim of the simulations is to find the correct parameters to mill the silver layer without inducing damage inside the active region of the device. The ion-solid interaction is theoretically simulated by a static interaction simulative software called SRIM, which considers the Stopping and Range of Ions in Matter (SRIM), [23,24]. In Figure 1a, the theoretical collision cascade of the gold double-charged ions at $35 \mathrm{keV}$ in a silver layer is shown. The collision cascade exhibits a box shape (blue dotted line) that is close to the ideal configuration, with a sputter yield of 15.6 atoms per incident ion. The sputter yield is a fundamental parameter for FIB patterning because it indicates the number of sputtered atoms per each incident ion; hence, the higher it is, the higher the number of removed atoms is, and therefore, the faster the patterning process is. The sputter yield is also necessary to calculate the dose, i.e., the charge left on the sample by the ion beam per centimeters square, which is usually expressed by $\mu \mathrm{C} / \mathrm{cm}^{2}$. The dose not only depends on the target material and the ion beam species but also on the thickness of the material to be milled [22]. To calculate the dose that is required, the following formula is used:

$$
\phi=\frac{\rho z}{Y}
$$


where $\phi\left(\mu \mathrm{C} / \mathrm{cm}^{3}\right)$ is the dose that is necessary to mill the thickness $z(\mathrm{~nm}), Y$ is the sputter yield (atoms/ions), and $\rho$ is the volumetric density of the target material in $\left(\right.$ atoms $\left./ \mathrm{cm}^{3}\right)$.

\subsection{Finite-Difference Time-Domain Simulations}

A schematic cross section of the proposed structures is shown in Figure 1b; the silver nanoarrays were selected because they allow the hybrid opto-plasmonic resonances in the NIR to be exploited as a result of the spectral matching between the surface plasmon polaritons at the metal-dielectric interface and the Rayleigh anomaly [26].

Therefore, the optimization of the geometrical parameters of the nanoarrays to enhance absorption in the NIR range inside of the photodetector are run by FDTD simulations [25]. In Figure 1c, an overview of the simulations run to the optimization of the nanoarray structure is shown. The aim of the simulations in Figure 1c is to determine the best metal thickness values (dot height, $x$-axis) and periodicity (pitch, $y$-axis) to have the highest quantum efficiency (QE) at $950 \mathrm{~nm}$, i.e., the highest number of collected electrons over the number of incident ions.

In Figure 1c, the QE that is shown is normalized with respect to the maximum QE value that was obtained by coupling the same Si photodetector to a perfect anti reflective coating (PARC), i.e., a stack of different material layers with a tuned refractive index that is able to almost null the fraction of the reflected light incident on the device. The simulations highlight four metal thickness couples and pitch values:

The values that are reported in Table 1 are referred to for both 1-dimensional (1D) and 2-dimensional (2D) arrays and have the following nomenclature: "p-xyz,h-abc", where $p$ stands for pitch and xyz are the values of the pitch in $\mathrm{nm}$, and where $\mathrm{h}$ stands for height and abc are the values of the metal thickness in $\mathrm{nm}$. The fabrication of the silver nanoarrays with the specific values reported in Table 1 presents some challenging features. For the nanoarrays with p-535,h-110 are the most replicable in both the $1 \mathrm{D}$ and 2D configurations 1. By contrary, the p-535,h-300 cannot be fabricated by FIB patterning, but the long dwell time (time spent by the ion beam on each point to mill the material) that is necessary to mill a $300 \mathrm{~nm}$ silver thickness would lead to a (i) low lateral definition of the structures and to a (ii) patterning time that is too long. Hence, the configuration p-535,h-300 is not considered, and for the same reasons, the configuration p-260,h-300 is also not considered. Similar to this is the fabrication of p-260,h-110, which presents some tricky features because of the high aspect ratio and the low beam spot size that is required. For the above-cited reasons, only the configuration with the lower metal thickness (p-535,h-110 and p-260,h-110) are considered for both the 1-D and 2-D configurations in this work. To characterize the electrical behavior of the enhanced detector, the device was electrically connected by gold wire bonding to a printed circuit board (PCB) presenting 24 pins: 20 for photodiode metal contact and 4 for reference.

Table 1. Reported couples of metal thickness and periodicity values at absorption peaks from Figure 1c.

\begin{tabular}{ccccc}
\hline & p-535,h-110 & p-535,h-300 & p-260,h-110 & p-260,h-300 \\
\hline Metal thickness (nm) & 110 & 300 & 100 & 300 \\
Periodicity (nm) & 535 & 535 & 260 & 260 \\
\hline
\end{tabular}

\section{Results and Discussion}

In the previous section, the results of the Montecarlo and FDTD simulations are reported and discussed. Hence, both the patterning parameters and geometry features are optimized to guarantee a working integrated Si-detector with a higher efficiency in the NIR region. The two configurations p-535,h-100-p-260,h-110 in both the 1-D and 2-D geometry were nanofabricated by means of the FIB technique with gold doubled-charged ions with an energy of $35 \mathrm{keV}$, a beam current of $19 \mathrm{pA}$, and a dose value that could be calculated by Equation (1). The SEM images of the patterned nanostructures are shown in Figure 2; the 1-D and 2-D nanoarrays that are at higher periodicity (p-535) and the 1-D 
nanoarray at smaller periodicity ( $\mathrm{p}-260)$ present better reproducibility and conformity. On the other hand, the 2-D nanoarrays at smaller pitch ( $\mathrm{p}-260)$ show a very low conformity in terms of the shape and dimension of the dots, which is probably the result of spot beam that was too big.

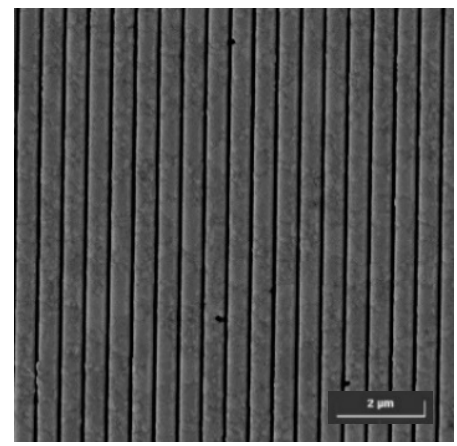

(a)

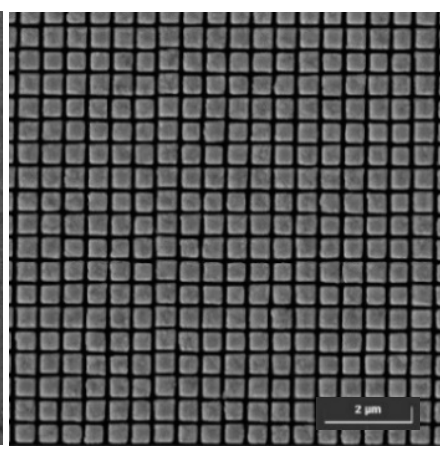

(b)

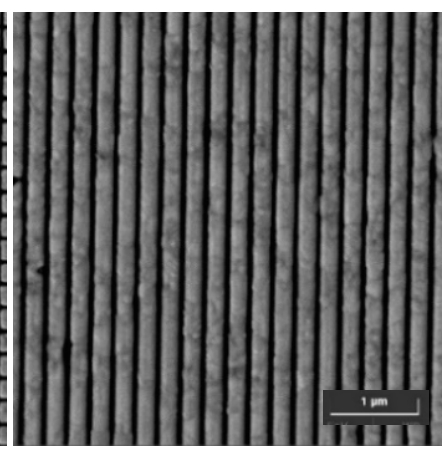

(c)

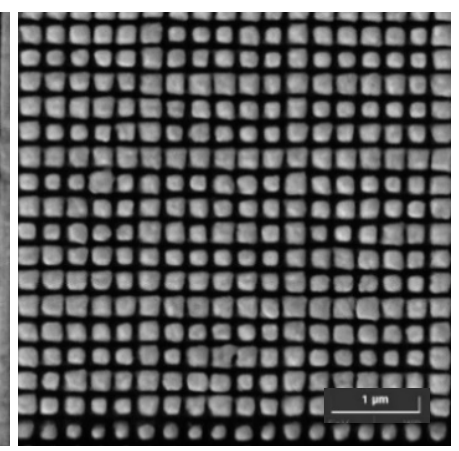

(d)

Figure 2. SEM images of the configuration (a) 1D p-535, (b) 2D p-535, (c) 1D p-260 and, (d) 2D p-260.

As discussed before, the main limitation of patterning nanostructures directly on an active device by means of FIB is the possibility of damaging the surface of the device, which is needed for ion implantation. Moreover, using gold ions as a beam species is a challenging choice because gold has been always considered to be a "silicon killer" ever since the early development of the silicon-based technologies, ref. [27]; therefore, even just a low concentration of gold ions inside could make the device could completely useless. The evaluation of the unaltered state of the device was assessed by dark current measurements, which refers to the current flowing inside the device under the conditions where there is no illumination. In a working silicon detector, the dark current, which is also called noise, should be at least three orders of magnitude less than the currents that are generated under illumination. The current that was generated under illumination had an intensity of hundreds of $\mu \mathrm{A}$ on average in the silicon photodetector produced in the Fondazione Bruno Kessler's facilities. In Table 2, the values of the dark current that were measured at the reverse applied voltage of $2 \mathrm{~V}$ for the four configurations shown in Figure 2 are reported. All four of the noise values are reported in the order of tens of $\mathrm{pA}$ and are comparable with the noise value of the reference diode (a not nanostructured diode). The dark currents reported in Table 2 are at least 5 orders of magnitude lower than the currents that are generated under illumination (hundreds of $\mu \mathrm{A}$ ). Therefore, gold ion-patterning silver directly on Si-photodiode does not damage the active behavior of the device, resulting in the integration being successful.

Table 2. Reported values of dark current of reference diodes (naked) and photodiodes patterned by FIB at $2 \mathrm{~V}$ in reverse mode.

\begin{tabular}{ccc}
\hline Reference & FIB 1D & FIB 2D \\
\hline \multirow{2}{*}{$21 \mathrm{pA}$} & $\mathrm{p}-\mathbf{5 3 5 , h}-\mathbf{1 1 0}$ & $\mathrm{p}-\mathbf{5 3 5 , h - 1 1 0}$ \\
& $41 \mathrm{pA}$ & $25 \mathrm{pA}$ \\
& $\mathrm{p}-\mathbf{2 6 0 , \mathbf { h } - 1 1 0}$ & $\mathrm{p}-\mathbf{2 6 0 , \mathbf { h } - \mathbf { 1 1 0 }}$ \\
& $10 \mathrm{pA}$ & $37 \mathrm{pA}$ \\
\hline
\end{tabular}

\section{Conclusions}

The ability to integrate plasmonic nanostructures directly on silicon photodiodes is of interest in many scientific and industrial domains, the reason for which is largely because of the unique optical properties that plasmonic structures can offer. Nevertheless, the characteristic size and the geometry of plasmonic structures are typically not of easy to fabricate. In this work, we demonstrated that the FIB technique is a solid solution that can 
be used for the nanopatterning of the plasmonic structures while they are directly on an active device through the optimization of the patterning parameters and the geometrical parameters by Montecarlo and FDTD simulations, respectively. In this contribution, we reported the successful integration of metallic plasmonic structures on a silicon photodiode without inducing any irreversible damage in the active region of the device. The results were demonstrated through the unaltered values of the dark current of the patterned diodes. This paves the way for further applications where plasmonic nanostructures can be integrated with photodetectors, such as all-in-one plasmonic biosensors.

Supplementary Materials: The poster presentation is available online at https: / / www.mdpi.com/ article/10.3390/ecsa-8-11259/s1.

Author Contributions: Conceptualization, G.P. and E.S.; methodology, E.S., G.P. and D.G.; software, E.S. and D.G.; validation, E.S., D.G. and G.P.; formal analysis, E.S. and A.C.; investigation, E.S., A.C. and D.G.; writing—original draft preparation, E.S.; writing—review and editing, L.P. and G.P.; visualization, A.C. and D.G.; supervision, G.P., D.G., L.P. and P.L.; project administration, G.P. and D.G.; funding acquisition, G.P. and D.G. All authors have read and agreed to the published version of the manuscript.

Funding: This project received funding from the EU-H2020 research and innovation programme under Grant Agreement 777222 ATTRACT project "PlaSiPM" and under grant agreement No 654360 NFFA-Europe.

Institutional Review Board Statement: Not applicable.

Informed Consent Statement: Not applicable.

Conflicts of Interest: The authors declare no conflict of interest.

\section{References}

1. Konstantatos, G.; Sargent, E.H. Nanostructured materials for photon detection. Nat. Nanotechnol. 2010, 5, 391-400. [CrossRef]

2. Soci, C.; Zhang, A.; Bao, X.Y.; Kim, H.; Lo, Y.; Wang, D. Nanowire photodetectors. J. Nanosci. Nanotechnol. 2010, 10, 1430-1449. [CrossRef] [PubMed]

3. Schuller, J.A.; Barnard, E.; Cai, W.; Jun, Y.C.; White, J.S.; Brongersma, M.L. Plasmonics for extreme light concentration and manipulation. Nat. Mater. 2010, 9, 193-204. [CrossRef] [PubMed]

4. Ghosh, B.; Espinoza-Gonzales, R. Plasmon-enhanced optical absorption with graded bandgap in diamond-like carbon (DLC) films. J. Mater. Sci. 2017, 52, 218. [CrossRef]

5. Gola, A.; Acerbi, F.; Capasso, M.; Marcante, M.; Mazzi, A.; Paternoster, G.; Piemonte, C.; Regazzoni, V.; Zorzi, N. NUV-Sensitive Silicon Photomultiplier Technologies Developed at Fondazione Bruno Kessler. Sensors 2019, 19, 308. [CrossRef] [PubMed]

6. Kim, C.-S.; Ahn, S.-H.; Jang, D.-Y. Review: Developments in micro/nanoscale fabrication by focused ion beams. Vacuum 2012, 86, 1014-1035. [CrossRef]

7. Wilhelmi, O.; Reyntjens, S.; Mitterbauer, C.; Roussel, L.; Stokes, D.J.; Hubert, D.H.W. Rapid Prototyping of Nanostructured Materials with a Focused Ion Beam. Jpn. J. Appl. Phys. 2008, 47, 5010-5014. [CrossRef]

8. Rubanov, S.; Munroe, P. The application of FIB milling for specimen preparation from crystalline germanium. Micron 2004, 35, 549-556. [CrossRef]

9. Schoendorfer, C.; Lugstein, A.; Bischoff, L.; Hyun, Y.; Pongratz, P.; Bertagnolli, E. FIB induced growth of antimony nanowires. Microelectron. Eng. 2007, 84, 1440-1442. [CrossRef]

10. Xu, X.; Wu, J.; Wang, X.; Zhang, M.; Li, J.; Shi, Z.; Li, H.; Zhou, Z.; Ji, H.; Niu, X.; et al. Ion-Beam-Directed Self-Ordering of Ga Nanodroplets on GaAs Surfaces. Nanoscale Res. Lett. 2016, 11, 38. [CrossRef]

11. Kawano, Y.; Fuse, T.; Toyokawa, S.; Uchida, T.; Ishibashi, K. Terahertz photon-assisted tunneling in carbon nanotube quantum dots. J. Appl. Phys. 2008, 103, 034307. [CrossRef]

12. Komiyama, S. Single-Photon Detectors in the Terahertz Range. IEEE J. Sel. Top. Quantum Electron. 2011, 17, 54-66. [CrossRef]

13. Pan, Z.W.; Dai, Z.R.; Wang, Z.L. Nanobelts of Semiconducting Oxides. Angew. Chem. Int. Ed. Engl. 1996, 291, 1947-1949. [CrossRef]

14. Iijima, S. Helical microtubules of graphitic carbon. Nature 1991, 354, 56-58. [CrossRef]

15. Takai, I.; Matsubara, H.; Soga, M.; Ohta, M.; Ogawa, M.; Yamashita, T. Single-Photon Avalanche Diode with Enhanced NIRSensitivity for Automotive LIDAR Systems. Sensors 2016, 16, 459. [CrossRef] [PubMed]

16. Niu, C.; Huang, T.; Hu, J. Plasmonic Nanograting Structures for Sensor Applications, Texas Symposium on Wireless and Microwave Circuits and Systems. In Proceedings of the Texas Symposium on Wireless and Microwave Circuits and Systems, Waco, TX, USA, 3-4 April 2014. 
17. Andersen, S.K.H.; Bogdanov, S.; Makarova, O.; Xuan, Y.; Shalaginov, M.Y.; Boltasseva, A.; Bozhevolnyi, S.I.; Shalaev, V.M. Hybrid Plasmonic Bullseye Antennas for Efficient Photon Collection. ACS Photon. 2018, 5, 692-698. [CrossRef]

18. Caldwell, J.D.; Glembocki, O.; Bezares, F.J.; Bassim, N.D.; Rendell, R.W.; Feygelson, M.; Ukaegbu, M.; Kasica, R.; Shirey, L.; Hosten, C. Plasmonic Nanopillar Arrays for Large-Area, High-Enhancement Surface-Enhanced Raman Scattering Sensors. ACS Nano 2011, 5, 4046-4055. [CrossRef]

19. Selvakumar, N.; Barshilia, H. Review of physical vapor deposited (PVD) spectrally selective coatings for mid- and hightemperature solar thermal applications. Sol. Energy Mater. Sol. Cells 2012, 98, 1-23. [CrossRef]

20. Stanley Middleman, B.; Hochberg, A.K. Process Engineering Analysis in Semiconductor Device Fabrication; Mcgraw-Hill College: New York, NY, USA, 1993.

21. Newman, T.H. High resolution patterning system with a single bore objective lens. J. Vac. Sci. Technol. B: Microelectron. Nanometer Struct. 1987, 5, 88. [CrossRef]

22. Orloff, J.; Utlaut, M.; Swanson, L. High Resolution Focused Ion Beams: FIB and its Applications. In High Resolution Focused Ion Beams: FIB and Its Applications; Springer Science and Business Media LLC: Berlin/Heidelberg, Germany, 2003.

23. Ziegler, J.F.; Biersack, J.P.; Ziegler, J.F. The Stopping and Range of Ions in Matter. In Treatise on Heavy-Ion Science; Bromley, D.A., Ed.; Springer: Boston, MA, USA, 1960.

24. Ziegler, J.F.; Ziegler, M.D.; Biersack, J.P. SRIM-The stopping and range of ions in matter. Nucl. Instrum. Methods Phys. Res. Sect. B Beam Interact. Mater. Atoms 2010, 268, 1818-1823. [CrossRef]

25. Lumerical: High-Performance Photonic Simulation Software. Available online: www.lumerical.com (accessed on 22 September 2021).

26. Maradudin, A.A.; Simonsen, I.; Polanco, J.; Fitzgerald, R.M. Rayleigh and Wood anomalies in the diffraction of light from a perfectly conducting reflection grating. J. Opt. 2016, 18, 024004. [CrossRef]

27. Davis, G.L. Gold in semiconductor technology. Gold Bull. 1974, 7, 90-96. [CrossRef] 